\title{
25Gb/s 3-level Burst-Mode Receiver for High Serial Rate TDM-PONs
}

\author{
Xin Yin, Jochem Verbist, Timothy De Keulenaer, Bart Moeneclaey, Jochen Verbrugghe, Xing-Zhi Qiu and \\ Johan Bauwelinck \\ Ghent University, INTEC/IMEC, Sint-Pietersnieuwstraat 41, 9000 Gent, Belgium \\ E-mail address: xin.yin@intec.ugent.be
}

\begin{abstract}
We report the first 25Gb/s 3-level modulated BM-RX employing a 1/4-rate linear BM APD-TIA and a custom decoder IC. We successfully demonstrated burst-mode sensitivity of $-20.4 \mathrm{dBm}$ with $18 \mathrm{~dB}$ dynamic burst-to-burst for $25 \mathrm{~Gb} / \mathrm{s}$ upstream links.

OCIS codes: (060.2360) Fiber optics links and subsystems; (060.0060) Fiber optics and optical communication.
\end{abstract}

\section{Introduction}

Access network infrastructure plays a key role in fulfilling the ever-increasing traffic demand and emerging service requirement. Among those solutions, passive optical networks (PONs) are being widely recognized as the most costeffective technology for access networks. Various time division multiplexing PON (TDM-PON) standards, e.g. GPON and GE-PON, have been proposed by two standard bodies, international telecommunications union (ITU) and institute of electrical and electronics engineers (IEEE) respectively. Meanwhile upstream burst-mode receivers (BM-RX), the most critical physical media dependent (PMD) component of PON systems, have been recently developed up to $10 \mathrm{~Gb} / \mathrm{s}[1,2]$ in parallel with both of the standardization processes.

Due to technology and cost challenges associated with further increasing the serial date rate, current NG-PON2 standard from ITU focuses the development on a time and wavelength division multiplexed (TWDM) PON, i.e., by basically stacking $10 \mathrm{~Gb} / \mathrm{s}$ XG-PONs at 4 or 8 wavelengths. Still, a low-cost single-wavelength upstream beyond $10 \mathrm{~Gb} / \mathrm{s}$ has the potential to serve as a per-wavelength upgrade path for NG-PON2, in line with recent researches on $40 \mathrm{~Gb} / \mathrm{s}$ high serial rate downstream for PONs $[3,4]$.

In this paper we present a first $25 \mathrm{~Gb} / \mathrm{s} 3$-level modulated BM-RX for high serial rate TDM-PONs. Instead of using non-return to zero (NRZ) format, 3-level BM detection is proposed by employing a 1/4-rate APD-based linear BM trans-impedance amplifier (TIA) and a custom duo-binary decoder chip. The proposed 25Gb/s BM-RX relaxes the bandwidth requirement on optical components, esp. the high-speed APDs, and can support a high loss budget of 25.4dB without optical pre-amplification.

\section{25Gb/s 3-level modulated BM-RX}

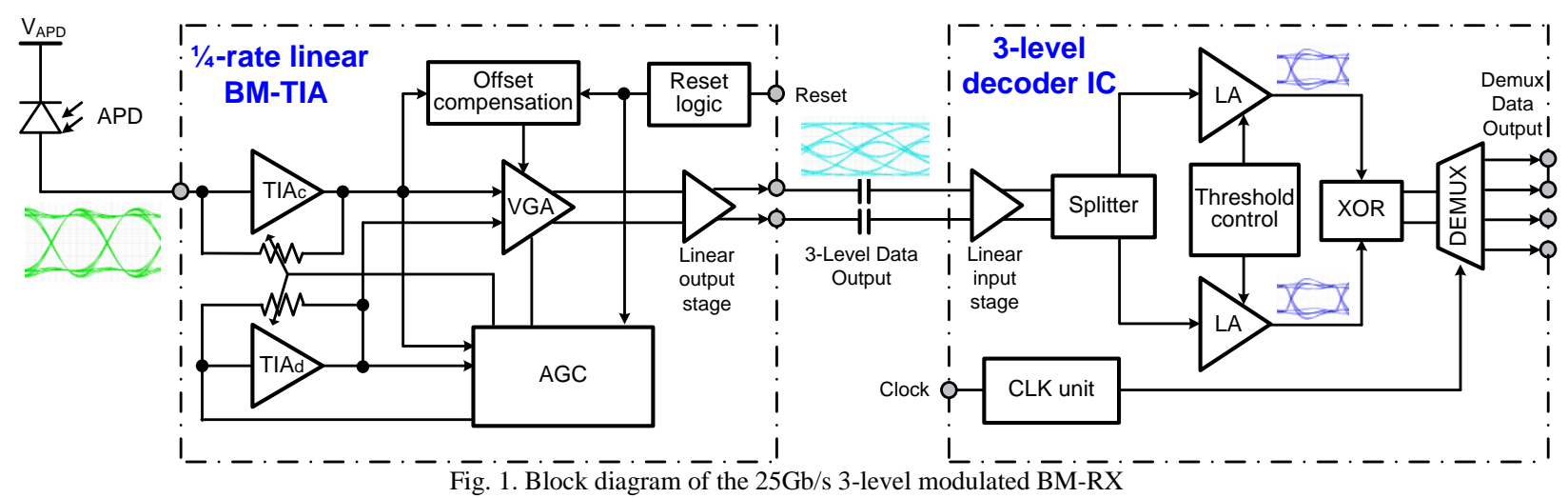

Figure 1 shows the block diagram of the proposed 25Gb/s BM-RX. The BM-RX consists of two blocks: a linear BM APD-TIA and a 3-level duo-binary decoder IC. Both blocks were designed and fabricated in a $0.13 \mu \mathrm{m}$ SiGe BiCMOS process. The linear BM-TIA is based on the design in [5] but with a trans-impedance gain adapted for the decoder IC and a fine-tuned bandwidth (one-fourth of the upstream data rate). The 1/4-rate small-signal bandwidth and linear operation are required for optimal 3-level signal generation. The linear BM-TIA consists of a core TIA TIAc, a dummy TIA TIAd, a variable gain amplifier (VGA), and a linear output stage. At the start of each burst, the automatic gain control (AGC) senses the output amplitude of the TIAc core and set its transimpedance gain within 10ns. The linear BM-TIA also performs a fast offset compensation process. This minimizes the DC offset in the 
output 3-level signal, and helps reduce the settling time of the decoder IC. The output of the linear BM-TIA is a 3level current-mode signal and ac-coupled to the 3-level decoder IC.

The subsequent custom decoder IC combines a 3-level signal decoding function and a 1 to 4 deserializer. It first amplifies the input signal linearly to partly compensate the internal loss of the RF splitter. After the splitter the upper and lower eyes are separated and amplified to their logic levels by two limiting amplifiers (LA). No threshold feedback has been realized in this first version decoder IC, so a feed-forward threshold control was implemented instead. The upper and lower logic signals then enter a high-speed digital XOR gate to regenerate the data in NRZ format. Finally the received NRZ data is deserialized into 4 CML outputs for interfacing with lower-speed components off-chip, e.g. FPGAs.

\section{Experimental setup and results}

The performance of the 3-level APD BM-RX was evaluated using the $25 \mathrm{~Gb} / \mathrm{s}$ experimental set-up as shown in Fig. 2. Two $1.3 \mu \mathrm{m}$ burst-mode transmitters (BM-TXs) named TX \#1 and TX \#2, are alternately sending burst packets. At the OLT, the linear BM APD-TIA was integrated with the 3-level decoder IC and the upstream BER was measured at one of the $4 \mathrm{CML}$ output channels at $6.25 \mathrm{~Gb} / \mathrm{s}$. Finally two branches of BM-TXs were built to emulate the worst TDMA scenario: a strong burst followed by a weak burst with a short guard time. The output optical power of the two BM-TXs can be adjusted by two variable optical attenuators (VOAs), namely VOA \#1 and VOA \#2 for respectively TX \#1 and TX \#2. A gated semiconductor optical amplifier (SOA) was used to increase the optical output power of the TX \#2 to $+5 \mathrm{dBm}$, in order to generate a sufficiently large loud/soft ratio for this experiment. A $10 \mathrm{~nm}$ coarse wavelength division multiplexing (CWDM) filter was used to remove out-of-band amplified spontaneous emission (ASE) noise from the SOA. The two TX outputs are combined by a $2 \times 2$ splitter, and fed to the BM-RX.

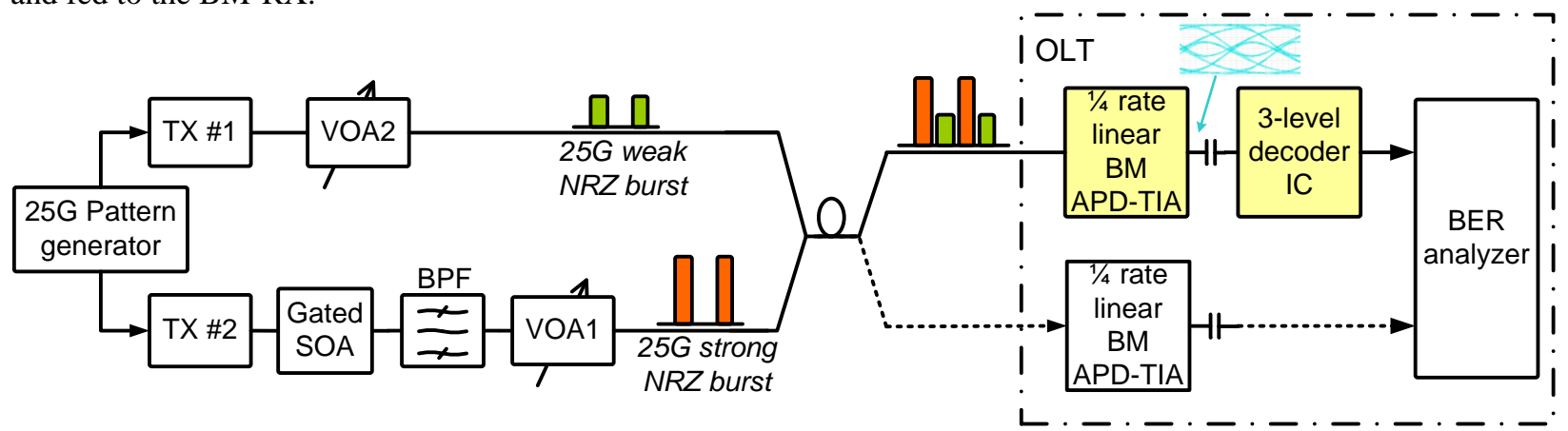

Fig. 2. Experimental setup used to characterize the BM-R $\bar{X}$

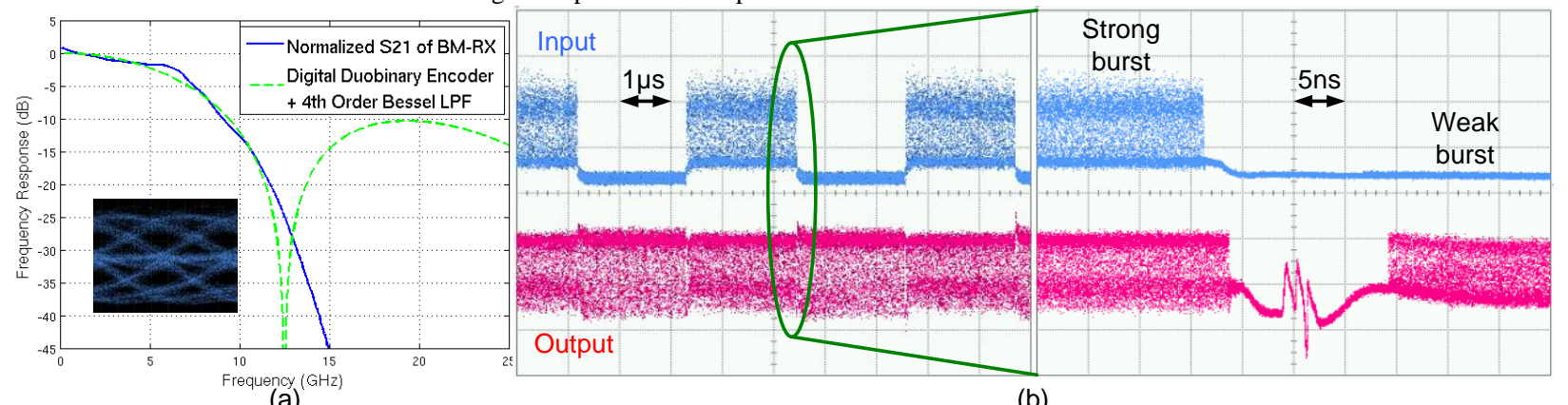

(a)

(b)

Fig. 3. (a) Measured RF frequency response and $25 \mathrm{~Gb} / \mathrm{s}$ eye-diagram (b) input and output waveforms of the BM APD-TIA

To generate 3-level modulation, the linear BM-TIA should have a bandwidth of roughly one-fourth of the data rate, i.e., $\sim 6.25 \mathrm{GHz}$ for $25 \mathrm{~Gb} / \mathrm{s}$. We measured the differential $\mathrm{O} / \mathrm{E}$ response of the linear BM APD-TIA assembly with a lightwave analyzer and it shows a $3 \mathrm{~dB}$ bandwidth of $6.7 \mathrm{GHz}$. The measured BM-TIA S21 response is plotted in Fig. 3(a), together with simulated frequency response of cascading a digital duobinary encoder and a $4^{\text {th }}$-order half-rate Bessel LPF. Both frequency responses agree nicely in the roll-off shape up to the Nyquist frequency. The measured 25Gb/s eye-diagram (Fig. 3(a)) at the output of the BM-TIA shows clearly the 3-level signal with two open eyes. Fig. 3(b) shows the measured received bursts and the output signal of the BM APD-TIA. The applied $25 \mathrm{~Gb} / \mathrm{s}$ burst packets consist of a $245 \mathrm{~ns}$ preamble and a $1800 \mathrm{~ns}$ payload of $2^{7}-1$ pseudo random bit sequence (PRBS) patterns. The guard time between bursts is set to 15ns. As shown in Fig. 3(b) the received weak burst following a strong burst has been recovered within $\sim 10 \mathrm{~ns}$. 
The measured B2B and BM BER curves are presented in Fig. 4. The APD multiplication factor M was set to $\sim 7$. The linear BM APD-TIA was initially characterized with a NRZ signal in a back-to-back (B2B) configuration for various data rates. The BM APD-TIA cannot directly support $25 \mathrm{~Gb} / \mathrm{s}$ NRZ without equalization. We measured the BER curves for $10 \mathrm{~Gb} / \mathrm{s}, 15 \mathrm{~Gb} / \mathrm{s}$ and $18 \mathrm{~Gb} / \mathrm{s}$ NRZ, and we found that there was $\sim 9.4 \mathrm{~dB}$ penalty at $18 \mathrm{~Gb} / \mathrm{s}$ with respect to the $10 \mathrm{~Gb} / \mathrm{s}$ case for a pre-FEC BER of $1 \mathrm{E}-3$. We then measured the BER performance of the proposed 3level modulation in both B2B and BM scenarios. The measured 25Gb/s B2B sensitivity at a pre-FEC BER of 1E-3 is $-22.4 \mathrm{dBm}$ and the sensitivity at a BER of $1 \mathrm{E}-10$ is $-16.3 \mathrm{dBm}$. We took the pre-FEC threshold of $\sim 1 \mathrm{E}-3$ as the reference because FEC coding is mandatory in NG-PON2 systems in order to maintain its compatibility with existing ODNs. With respect to $18 \mathrm{~Gb} / \mathrm{s}$ NRZ transmission, the $25 \mathrm{~Gb} / \mathrm{s} 3$-level detection experiences a sensitivity improvement of $3.6 \mathrm{~dB}$, which is primarily explained by the reduced $18 \mathrm{~Gb} / \mathrm{s}$ NRZ eye openings due to the higher bandwidth requirement. With two branches of BM-TXs, the BM-RX sensitivity measured on the weak packet emitted by BM-TX \#1 was $-22.4 \mathrm{dBm}$ with a static optical power level (0dB loud/soft). The BM-RX was also assessed in different loud/soft ratios and the measured BM-RX penalties due to the preceding loud burst are shown in Fig. 4(b). There was $\leq 0.6 \mathrm{~dB}$ penalty for a loud/soft ratio up to $10 \mathrm{~dB}$. The BM penalty was $2 \mathrm{~dB}$ for the maximum loud/soft ratio of $18 \mathrm{~dB}$.

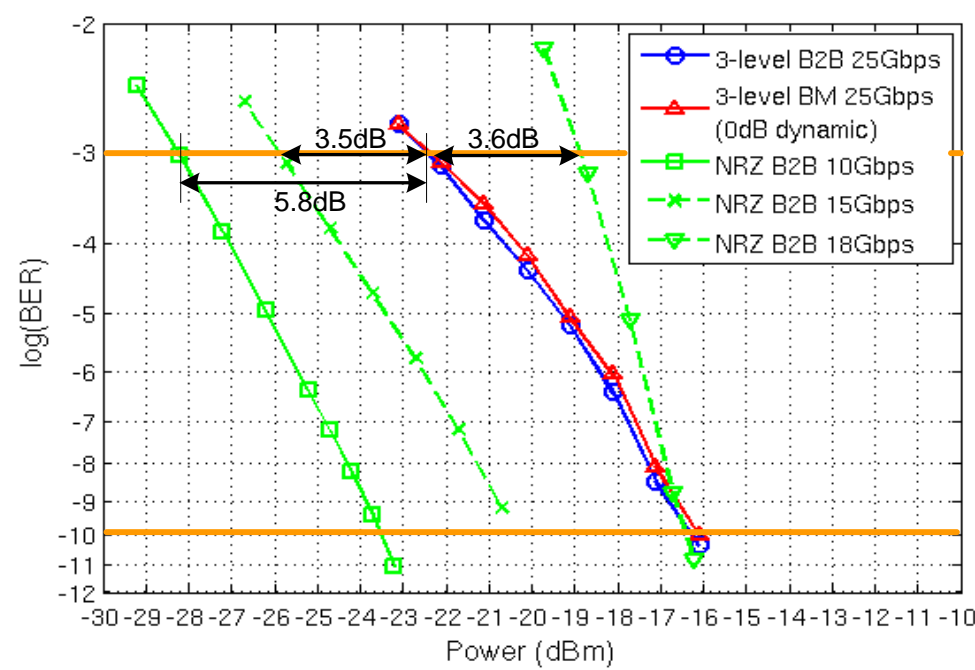

(a)

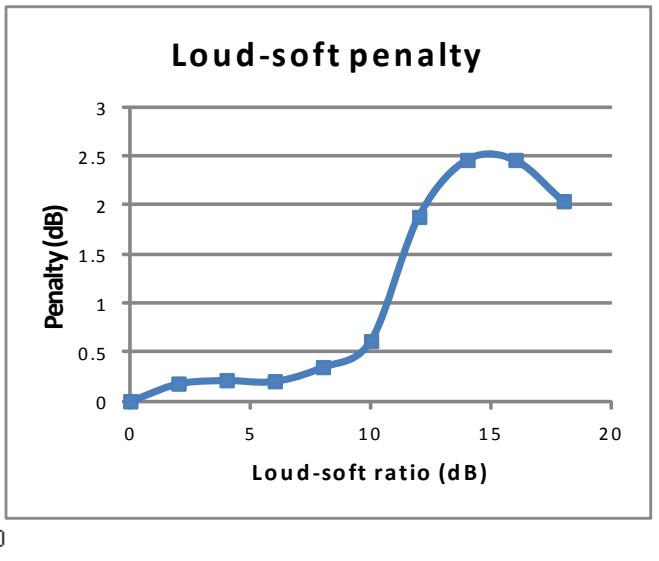

(b)

Fig. 4. (a) Comparison of BER between NRZ and 25Gb/s 3-level modulation (b) BM loud-soft penalty

\section{Conclusions}

We presented, for the first time, a 25Gb/s upstream BM-RX that utilizes 3-level detection at OLTs. Using a low-cost 1/4-rate linear BM APD-TIA and a custom decoder IC, it achieved an excellent RX sensitivity of $-22.4 \mathrm{dBm}$ without optical pre-amplifier or power-hungry DSP. In the worst case of $18 \mathrm{~dB}$ power difference between strong and weak bursts, we successfully demonstrated BM sensitivity of $-20.4 \mathrm{dBm}$ and a loss budget of $25.4 \mathrm{~dB}$ is therefore compatible for $25 \mathrm{~Gb} / \mathrm{s}$ upstream in high serial rate TDM-PONs.

\section{Acknowledgement}

The authors would like to thank the support from the European Union FP7 under grant agreement n. 318137 (Collaborative project "DISCUS"), IWT, Special Research Fund of Ghent University, and Sumitomo Electric Devices Innovations, Inc for their professional linear BM APD-TIA Assembly.

\section{References}

[1] M. Noda, N. Suzuki , S. Yoshima, M. Nogami and J. Nakagawa, "Technology progress of high-speed burst-mode 3R receiver for PON applications," in Proceeding of Optical Fiber Communication Conference (OFC), (OSA, 2012), paper OTh4G.6.

[2] X. Z. Qiu, X. Yin, J. Verbrugghe, B. Moeneclaey, A. Vyncke, C. Van Praet, G. Torfs, J. Bauwelinck, and J. Vandewege, "Fast synchronization 3R burst-mode receivers for passive optical networks [invited tutorial]," JLT 32, 644-659 (2014).

[3] X. Yin, X. Z. Qiu, G. Torfs, C. Van Praet, R. Vaernewyck, A. Vyncke, J. Verbrugghe, B. Moeneclaey, M. Ruffini, D. B. Payne, and J. Bauwelinck, "Performance evaluation of single carrier 40-Gbit/s downstream for long-reach passive optical network," in Proceeding of 18th International Conference on Optical Network Design and Modeling (ONDM), (IEEE, 2014), pp. 162-167.

[4] D. van Veen, V. Houtsma, A. Gnauck, and P. Iannone, "40-Gb/s TDM-PON over 42 km with 64-way Power Split using a Binary Direct Detection Receiver," in Proceeding of 40th European Conference on Optical Communication (ECOC), (OSA, 2014), paper PD.1.4.

[5] X. Yin, B. Moeneclaey, X. Qiu, J. Verbrugghe, K. Verheyen, J. Bauwelinck, J. Vandewege, M. Achouche, and Y. Chang, "A 10Gb/s APDbased linear burst-mode receiver with 31dB dynamic range for reach-extended PON systems," Optics Express 20, B462-B469 (2012). 